\title{
Stratégies et tendances de communication dans les entreprises chiliennes
}

Communication strategies and tendencies in Chilean firms

Lucía Castellón et Francisco Javier Mas

\section{(2) OpenEdition}

\section{Journals}

Édition électronique

URL : http://journals.openedition.org/communicationorganisation/4636

DOI : 10.4000/communicationorganisation.4636

ISSN : $1775-3546$

Éditeur

Presses universitaires de Bordeaux

\section{Édition imprimée}

Date de publication : 1 juin 2014

Pagination : 271-280

ISBN : 978-2-86781-904-9

ISSN : 1168-5549

Référence électronique

Lucía Castellón et Francisco Javier Mas, « Stratégies et tendances de communication dans les entreprises chiliennes », Communication et organisation [En ligne], 45| 2014, mis en ligne le 01 juin 2017, consulté le 21 décembre 2020. URL : http://journals.openedition.org/

communicationorganisation/4636; DOI : https://doi.org/10.4000/communicationorganisation.4636

(C) Presses universitaires de Bordeaux 


\title{
Stratégies et tendances de communication dans les entreprises chiliennes
}

\author{
Lucía Costellón' et Francisco Javier Mas²
}

Avec le concours de N. D’Almeida

\section{Présentation}

Ces vingt dernières années représentent une période de profonds changements pour la société chilienne dans son ensemble. Plusieurs processus se déroulent en parallèle et permettent d'envisager trois modes de transformation :

a) Sur le plan culturel, un changement abrupt a eu lieu nous faisant passer d'une société moderne ayant de profonds ancrages idéologiques à une société postmoderne marquée par la diversité ;

b) Sur le plan politique, un long processus de transition d'une dictature vers la démocratie s'est effectué, marquant l'affirmation d'une opinion publique ;

c) Sur le plan de la communication, nous avons été témoin d'une révolution digitale ayant de grandes répercussions depuis l'installation de nouvelles technologies de l'information et de la communication et du lent processus de consolidation de la société de l'information dans le pays vers un horizon chaque fois plus globalisé dans les domaines économique, politique et culturel.

Une industrie de la communication se développe, caractérisée par une forte croissance. Ceci vaut dans le secteur des entreprises où se créent des services visant à améliorer la réputation corporate des entreprises, la formation des cadres aux situations de crise jusqu'au marketing et aux communications internes.

1 Lucía Castellón Aguayo est actuellement Directrice de L'Institut de Communication et Nouvelles technologies de 1'Université Mayor de Santiago du Chili. Lucia Castellon a créé en 1988 L'École de Journalisme à l'Université Diego Portales, université dont elle a été doyenne pendant plusieurs années. Personnalité fondatrice des formations en journalisme et communication au Chili elle est un acteur important des associations de recherche sud américaines : vice-présidente d'IBERCOM puis d'ALAIC. Elle a créé et porté la première chaire UNESCO dans le domaine communication au Chili ; Lucia.castellon@umayor.cl

2 Francisco Mas, Professeur Titulaire et Maître de Recherches en Communication et Nouvelles Technologies et Communication Stratégiques et Gestion de Marque à l'Université Mayor de Santiago du Chili. Francisco a obtenu il y a deux ans le doctorat en Communication et Organisation à l'Université de Málaga, Espagne. Sa formation antérieure est marquée par la littérature (maîtrise en Lettres de l'Université Catholique du Chili) et le journalisme (Licenciature obtenue à 'université Diego POrtales) ; francisco.mas@umayor.cl 
À la fin des années 80, le Chili a vécu un processus de changement politique profond. Il laisse derrière lui la dictature militaire et s'insère dans les marchés internationaux par le biais de divers traités de libre-échange parallèles à l'instauration de la démocratie. Ces changements internes et externes, au Chili, se sont répercutés sur les organisations publiques et privées, et principalement sur les entreprises qui ont dû, pendant la consolidation du modèle de libre-échange néolibéral des gouvernements en concertation avec les partis politiques, s'aligner à des niveaux de compétitivité jamais vus auparavant dans le pays. Ceci conduit lentement les entreprises à considérer que les intangibles étaient les actifs les plus importants sur lesquels elles comptaient, tout en comprenant que leur gestion était fondamentale pour leurs exercices et investissements futurs. Ainsi, la demande de services de stratégie de communication s'est accrue de façon exponentielle dans le pays afin de consolider ces entreprises sur le marché national et aussi afin de les positionner sur les marchés internationaux exigeants et complexes.

Ces dernières années, l'industrie de la stratégie de communication a présenté des indices élevés de croissance. Elle est marquée par le développement d'une offre de services chaque fois plus diversifiée qui englobe tour à tour la conception, la planification stratégique, la gestion, le contrôle et l'évaluation des processus de communication autant interne qu'externe des entreprises.

Les grandes transformations dans les économies globalisées et les marchés qui sont chaque fois plus sophistiqués et concurrentiels, génèrent, au Chili, un développement accéléré du secteur de la stratégie de communication. La bataille pour le positionnement de l'entreprise auprès des consommateurs et autres publics stratégiques pour l'entreprise, est chaque fois plus difficile. Aussi les acteurs ont du s'adapter aux changements constants du marché, à une scène globalisée et à des marchés toujours plus interdépendants, à des innovations technologiques liées à la gestion du commerce.

La stratégie stratégique, dans le pays, acquiert une importance toujours plus grande dans la mesure où les entreprises ont découvert à partir d'autres expériences le besoin de cette stratégie pour aligner toute l'entreprise avec ses stratégies commerciales. Les tendances internationales, principalement anglosaxonnes et européennes en relation avec de bonnes pratiques de commerce supposent des compétences de gestion, vitales pour le développement de la communication stratégique dans les entreprises. Les facteurs considérés comme essentiels peuvent être ainsi présentés : à savoir la cohérence entre le discours déclaré et les actions effectuées ; la vision, la mission et les objectifs clairement déterminés et assumés par l'entreprise, et les moyens de dialogue avec tous leurs publics (Sylvestre et alt. 2009).

C'est dans cette perspective que les entreprises nationales sont aujourd'hui fortement conscientes du caractère déterminant du contexte globalisé qui suscite de manière chaque fois plus complexe et intégrée, le développement de la gestion de communication corporate. Compter sur des stratégies chaque 
fois plus intégratrices des modèles d'affaires des entreprises avec leurs publics stratégiques variés est devenu un besoin fort et un élément de différenciation pour le succès des entreprises, pour leur survie face à la concurrence et leur croissance économique future.

\section{L'industrie de la communication stratégique}

Le marché de la stratégie de communication, tant celui de l'offre que celui de la demande, est de plus en plus sophistiqué (Herrera R, 2010). Du point de vue de l'offre, Herrera $\mathrm{R}$, dans son article intitulé "Croissance et développement de l'industrie de la stratégie de communication au Chili ", publié dans la revue Chasqui n ${ }^{\circ} 108$, écrit qu'elle est constituée par trois grands acteurs : a) les entreprises de communication ; b) les agences de publicité ; c) les entreprises de marché. Pour sa part, la demande est constituée non seulement par les entreprises privées mais aussi par les entreprises publiques et les organisations non-gouvernementales.

Dans l'ensemble, le marché offre seize types d'activités et de services et 26 modes d'approche essentiellement centrées sur "les études de marque, de l'image, de la réputation, de la satisfaction des clients, de l'évaluation publicitaire, de l'opinion, des médias, des multimédias, de la loyauté, de la communication interne, de la relation avec les clients, des tendances, des études sociales, de l'éducation, de la société, de l'économie, RSE, des talents, du climat et de l'ethnographie » (Herrera R, 2010 : 64).

Cette première étude rigoureuse du marché chilien de la stratégie de communication menée par Herrera souligne que cette industrie se chiffre à plus de 94 millions de dollars par an et que ce sont les entreprises ou sociétés de conseil en stratégie de communication qui sont les acteurs majeurs de l'offre de services (au détriment des agences de publicité).

Par ailleurs, la demande de communication est fournie par un ensemble de grandes entreprises lequel est le plus grand consommateur de services de communication qui constitue un marché de 8025 entreprises. On peut remarquer que seulement $40 \%$ des grandes entreprises ont un responsable qui dirige la communication au niveau stratégique. Les $60 \%$ restants possèdent un niveau opérationnel ou n'ont pas de département de communication dans leurs entreprises. Environ les $90 \%$ des directions des grandes entreprises qui dirigent la communication ne possèdent pas de formation dans ce domaine stratégique. La demande des entreprises du secteur public est chaque fois plus pertinente pour ce marché et leurs plus grands consommateurs sont les pouvoirs de l'État chilien. En ce qui concerne les organisations nongouvernementales, la demande est croissante également car elles doivent se rendre visibles pour concourir à l'obtention des ressources qui sont assignées au secteur du marché social (Herrera R, 2008). 


\section{Tendances de stratégie de communication dans la gestion de marque}

Comprendre l'état actuel du branding dans notre pays suppose de comprendre les marques dans leur contexte. Au Chili, la gestion de marque dans les entreprises a été très importante ces dernières décennies. Néanmoins cette gestion dans les entreprises chiliennes n'a pas été uniforme et constante ces dernières années. Durant cette période, diverses modalités ou tendances ont été adoptées, certaines étant plus pertinentes, significatives et différentes que d'autres.

Les deux textes les plus intéressants qui illustrent ce cadre économique, social et culturel où se sont développées les marques, au Chili, ont été constitués dans les livres "Chile Marca Registrada " et "Marcas en la Cabeza " publiés respectivement en 2008 et en 2009 par Pedro Alvarez et Eduardo Carrasco. Les deux textes abordent la dimension historique des marques chiliennes à partir du contexte historico-social, lié à la scène économique établie lors de l'instauration de la république au $\mathrm{XX}^{\mathrm{e}}$ siècle, qui a fluctué entre un État bienfaiteur et protectionniste et son déplacement actuel vers le modèle de marché.

Les marques chiliennes doivent être comprises dans leurs processus historiques et sociaux ce qui permet d'établir certaines références minimales pour comprendre le devenir des marques au Chili et les tendances de leur stratégie communicationnelle sachant que pour ce dernier aspect, il est important de comprendre que ce sont les propres marques qui ont construit les dialogues pour se lier à leurs audiences avec les moyens suivants par exemple : transmettre l'information, utiliser des dispositifs de persuasion et d'identification sociale ou tout simplement les utiliser comme moyen d'évocation (Carrasco E, 2009).

Selon Alvarez P, 2008, les marques au Chili, à partir de cette période, peuvent être situées et caractérisées clairement depuis l'accès aux données issues de l'information écrite disponible dans les médias nationaux et la documentation visuelle provenant de différentes sources : registres de marques commerciales et patentes du Département de Propriété Industrielle ; archives de certaines entreprises et sociétés anonymes ; collections particulières et publicité de produits de consommation et d'usage quotidien dans divers médias et supports.

De cette manière, les marques ont suivi une évolution caractérisée par trois stades qui vont des origines de l'industrialisation au Chili et qui montrent comment la production d'articles en série a été définie par une nouvelle sphère d'échange social où les liens communautaires basés sur les traditions locales, ont été lentement érodés par la logique de marché, dans un contexte d'affaiblissement croissant des codes nationaux anciens qu'ils soient éthiques, politiques ou religieux. 
Ces processus peuvent être résumés en trois grandes phases historiques : a) la construction de la République ; b) la consolidation de l'État chilien ; c) le modèle néolibéral du marché à partir de la dictature militaire dans les années 70 .

La première phase est spécifique car la marque est alors construite sur l'image et les ressemblances des marques adoptées par les élites. Elle est formée par des représentations commerciales façonnées autour d'articles d'usage quotidien qui éprouvent le besoin impérieux de construire un imaginaire républicain tel qu'il existait dans les principales capitales européennes de l'époque, tout en ignorant le passé indigène et l'héritage espagnol spécifiques du Chili. Elle se caractérise principalement par les «symboliques et héraldiques » provenant de la tradition médiévale européenne ; gestes épiques ; personnalités éminentes et héros.

La deuxième phase concerne l'étape de la consolidation de l'État chilien où les marques avancent parallèlement avec l'émergence des médias de communication de masse et l'hégémonie de l'État en tant qu'agent productif et levier de dynamisme économique et social.

La troisième et dernière phase, et sans aucun doute la plus complexe et pertinente, est formée par notre société contemporaine caractérisée par le modèle de marché néolibéral qui établit que les marques se situent dans un référent identitaire transversal à la société chilienne : la consommation.

Selon Tironi, E et Cavallo, A. 2004, la stratégie de communication est récemment comprise, au Chili, comme un outil disponible pour les organisations de diverses natures, et se réfère à la gestion intégrale ${ }^{3}$ de la marque des organisations. Selon ces auteurs, la stratégie de communication agit pour réunir et systématiser le capital de prestige et de crédibilité dont toute organisation a besoin pour réussir ses objectifs et faire face aux tensions et aux crises de nos sociétés. À partir de cette définition, nous pouvons établir que la mission de la stratégie de communication est de projeter l'identité des organisations en une image qui suscite la confiance auprès de son entourage pertinent et suscite l'adhésion à ses objectifs rendus publics.

La stratégie de communication réunit et articule d'autres pratiques comme le journalisme, la publicité, les relations publiques, l'organisation de la communication et le marketing, son centre principal étant l'organisation entière et son positionnement. Ainsi à partir des observations des stratégies de communication des entreprises chiliennes, de leurs pages web, de leurs spots publicitaires graphiques, sonores et audiovisuels, de leurs actions marketing, relations publiques, sondages online, managements de branding, marketing et études, il a été possible de déterminer que les tendances de stratégies de communication de gestion de marques présentes sur le marché de la communication d'entreprises chiliennes sont principalement les suivantes :

- la gestion de son identité- image- réputation corporate;

3 Au double sens de complète et intégrée. 
- Le social Branding ou communication de responsabilité sociale d'entreprises - RSE- ;

- le Cobranding permettant de lier plusieurs marques en un même message, le Green Branding ; e) Branding Mutante, l'Antibrand, le Branding 2.0 et le Branding Experienciel.

Ces tendances ont été, dans une première étape, étudiées de manière exploratoire et descriptive, afin ensuite de comprendre ses éléments sémiotiques, narratifs et ses structures persuasives. Nos conclusions ont été obtenues sur la base de la méthode suivante : a) Analyse cyber graphique et bibliographique ; b) Étude de 150 sites web d'entreprises, consultants, agences de publicité et réseaux sociaux ; c) analyse de spots publicitaires, relations publiques et informations graphiques et audiovisuelles; entretiens complets et questionnaires online adressés à des cadres supérieurs, directeurs de société de conseils en communication stratégique, agences de publicité et professeurs universitaires de branding, marketing ou communication corporate, ce qui a mobilisé un échantillon de 40 individus pour chaque catégorie.

Une grande partie des efforts que les entreprises chiliennes poursuivent se situent dans le besoin de gérer la marque au sens corporatif. Même s'il existe des approches théoriques qui font état d'un actuel développement de ces activités, priment celles qui se centrent tant dans le composant de l'image comme dans l'identité au sein d'une constance qui n'est pas dichotomique en soi-même.

Bien que ces tendances peuvent s'identifier et se définir précisément, elles sont en fait le plus souvent associées à des référents théoriques bien délimités. Une partie de ces influences sont redevables d'au moins trois approches théoriques dans la conception de l'image, de l'identité et de la réputation corporate.

La première référence exprime une relation à ce qui (Costa J, 2003), se définit comme image corporate. Une grande partie de ce qui se fait en la matière est associée au concept d'image d'entreprise conçu comme représentation mentale, intervenant dans l'imaginaire collectif, constituée d'un ensemble d'attributs et valeurs qui fonctionnent comme un stéréotype et déterminent la conduite et opinons de cette collectivité. De cette façon, l'image corporate est dotée de certaines caractéristiques stratégiques qui permettent : de faire ressortir l'identité différenciée de l'entreprise, de définir le sens de la culture organisationnelle, de construire la personnalité et le style corporate, de renforcer l'esprit de corps et réorienter le leadership, d'attirer les meilleurs talents, de motiver le marché de capitaux; d'éviter les situations critiques; de développer des nouveaux produits et services; de relancer l'entreprise; de créer une opinion publique favorable à son égard; de réduire les messages involontaires ; d'optimiser les investissements en communication; d'accumuler réputation et prestige ; d'attirer et fidéliser les clients et d'inventer le futur. 
Une autre influence importante est celle (Capriotti P. 2009) qui établit une approche conceptuelle concernant le continuum identité - image - réputation corporative. Pour Capriotti, l'important est de référer cette question aux sujets qui interviennent dans le processus général de communication. Ainsi, dans ce processus général de la communication au sein des entreprises, l'élaboration et interprétation de l'information n'est pas, une action exclusive, que ce soit celle de l'émetteur - la construction - ou celle du récepteur - l'interprétation. Au contraire, la construction du sens est constituée par un continuum, celuici étant un procédé d'interactions mutuelles partagées entre elles. En fait, ce que les entreprises gèrent à travers la communication stratégique, c'est la tentative d'établir un dialogue, un contrat de sens partagé entre l'émetteur -l'entreprise- et certains destinataires - ses publics-, dans lequel une partie élabore et propose un sens à l'information (émetteur - entreprise) tandis que l'autre partie interprète et attribue un sens à l'information (destinataires publics) (Capriotti P. 2007).

Cette délimitation permet de comprendre deux dimensions pour lesquelles les entreprises chiliennes travaillent ce continuum d'une part comme un constructivisme d'émission où le sens est lié à l'émetteur ; d'autre part un constructivisme de perception dans lequel le sens est rapporté au récepteur. Ceci peut se résumer dans le cadre suivant :

\begin{tabular}{|l|l|l|l|}
\hline & \multicolumn{1}{|c|}{ Image } & \multicolumn{1}{c|}{ Positionnement } & \multicolumn{1}{c|}{ Reputation } \\
\hline $\begin{array}{l}\text { Constructivisme } \\
\text { d'émission }\end{array}$ & $\begin{array}{l}\text { Outils pour influer sur } \\
\text { les publics }\end{array}$ & $\begin{array}{l}\text { Outils pour influer sur } \\
\text { les consommateurs. }\end{array}$ & $\begin{array}{l}\text { Outils pour influer } \\
\text { sur les stakeholders. }\end{array}$ \\
\hline $\begin{array}{l}\text { Constructivisme } \\
\text { de Reception }\end{array}$ & $\begin{array}{l}\text { Associations dans } \\
\text { l'esprit des publics. }\end{array}$ & $\begin{array}{l}\text { Associations } \\
\text { dans l'esprit des } \\
\text { consommateurs. }\end{array}$ & $\begin{array}{l}\text { Associations } \\
\text { dans l'esprit des } \\
\text { stakeholders. }\end{array}$ \\
\hline
\end{tabular}

Source : Capriotti P, 2010

Enfin, il est important de noter qu'au Chili, durant ces dernières années, nous avons assisté à une prolifération de classements- rankings de marques de toutes natures, auxquels se soumettent les entreprises soucieuses de leur évaluation. Ces classements permettent de configurer l'état de la communication corporative et sa gestion stratégique et ils l'intensifient

À titre d'exemples, voici ci dessous une partie des principaux classements. Ce tableau fait aussi apparaître la méthodologie utilisée pour leur établissement et le lien étroit noué avec la presse qui les publie et les donne à voir. 


\begin{tabular}{|c|c|c|c|}
\hline Nom & Qui la realise & Qui la publie & Explication \\
\hline BAV & The Lab Y\& R & $\begin{array}{l}\text { Revue : Qué } \\
\text { Pasa. }\end{array}$ & $\begin{array}{l}\text { Mesure la valeur de marque à partir de } \\
4 \text { variables : relevance, différenciation, } \\
\text { estime et familiarité, à partir d'enquêtes } \\
\text { auprès des consommateurs. Il est } \\
\text { parfaitement reconnu et globalisé, } \\
\text { existe depuis plus de } 15 \text { ans au niveau } \\
\text { mondial et depuis } 10 \text { ans au Chili. }\end{array}$ \\
\hline $\begin{array}{l}\text { Ranking de } \\
\text { Reputation } \\
\text { Corporate }\end{array}$ & $\begin{array}{l}\text { HK Captiva - } \\
\text { Collect }\end{array}$ & $\begin{array}{l}\text { Quotidien : La } \\
\text { Tercera }\end{array}$ & $\begin{array}{l}\text { À partir de } 3500 \text { sondages de } \\
\text { consommateurs, sont évalués } 19 \\
\text { attributs, groupés en six dimensions : } \\
\text { émotionnelle, exercice financier, } \\
\text { responsabilité sociale, ambiance de } \\
\text { travail, gestion et leadership et produits } \\
\text { et services. Est développée au Chili } \\
\text { depuis } 2002 \text {. }\end{array}$ \\
\hline $\begin{array}{l}\text { Mesure } \\
\text { d'Entreprise } \\
\text { de } \\
\text { Réputation } \\
\text { Corporative } \\
\text { (Merco) }\end{array}$ & $\begin{array}{l}\text { Moniteur } \\
\text { D’Entreprise } \\
\text { de } \\
\text { Réputation } \\
\text { Corporative } \\
\text { (Merco) }\end{array}$ & $\begin{array}{l}\text { Quotidien : El } \\
\text { Mercurio }\end{array}$ & $\begin{array}{l}\text { A pour principe que mesurer la } \\
\text { réputation est différent que de } \\
\text { mesurer l'image. Pour arriver à ses } \\
\text { résultats, il comprend les valorisations } \\
\text { d'entrepreneurs, employés, syndicats, } \\
\text { consommateurs, experts ainsi que des } \\
\text { vérifications de données reçues. A été } \\
\text { réalisé pour la première fois cette année } \\
\text { au Chili. }\end{array}$ \\
\hline $\begin{array}{l}\text { Ranking de } \\
\text { RSE }\end{array}$ & PRO Humana & $\begin{array}{l}\text { Revue : Qué } \\
\text { Pasa }\end{array}$ & $\begin{array}{l}\text { Se divise en deux : l'évaluation } \\
\text { intégrale composée de } 109 \text { affirmations } \\
\text { alimentées au travers de vérificateurs } \\
\text { qui évaluent chaque politique de RSE. } \\
\text { L'enquête auprès des employés mesure } \\
\text { la culture interne de RSE à travers des } \\
\text { indicateurs d'évaluation, connaissance } \\
\text { et pratique. Les deux enquêtes ont } \\
\text { une pondération de } 50 \text { chacun. Pour } \\
\text { récompenser les entreprises les plus } \\
\text { responsables, sont retenues celles qui } \\
\text { obtiennent les meilleures moyennes } \\
\text { finales. Est réalisé depuis } 2005 \text {. }\end{array}$ \\
\hline $\begin{array}{l}\text { Cabinet de } \\
\text { Marques y } \\
\text { styles de vie, } \\
\text { Chile 3D }\end{array}$ & Collect & $\begin{array}{l}\text { Revue : Poder y } \\
\text { Negocios }\end{array}$ & $\begin{array}{l}\text { Enquête auprès de } 1280 \text { personnes, } \\
\text { et mesure le capital de marque à partir } \\
\text { du prestige, affection et présence. La } \\
\text { dernière publication date de décembre } \\
2009 \text {. }\end{array}$ \\
\hline
\end{tabular}

Source : Mas, Francisco. Tesina de Suficiencia Doctoral. Universidad de Málaga, España, 2010

On peut tirer deux conclusions générales de ces classements : Au delà de l'évaluation subjective qui peut se faire de ces études, toutes reposent sur des méthodologies valides, validées et rendues publique, de plus ils mobilisent d'importants niveaux de représentation de population ou d'échantillon d'enquête. Les deux environnements et préoccupations dans lesquels se 
développent les rankings sont la Valeur de Marque (que nous comprenons comme la capacité d'une marque à générer de la demande) et la Réputation de Marque (on inclut dans cette catégorie les rankings de RSE).

Par ailleurs et à partir des réponses obtenues à partir d'entretiens avec des cadres exécutifs du secteur du branding, marketing et communication corporate, nous notons que la tendance à vouloir organiser et gérer le continuum image - identité - réputation dans les entreprises, est pleinement vérifiée. Et ceci avec toujours plus d'intérêt, car $95 \%$ des cadres supérieurs consultés considèrent qu'il est trés important de gérer et positionner sa marque corporate. La vitalité de cette tendance de gestion de marque peut se vérifier dans les réponses que donnent aussi bien les directeurs de sociétés de conseil en communication stratégique que les principales et plus importantes agences de publicité du Chili. $92 \%$ des personnes interrogées considèrent qu'il est très important pour les marques de gérer et positionner leur dimension corporate en termes d'intérêt et d'impact auprès de leurs publics. La même réponse est faite par les professeurs universitaires de marketing-communication interrogés sur le niveau d'importance accordé aux marques qu'ils ont pu analyser professionnellement. $91 \%$ des réponses recueillies montrent qu'il s'agit d'une dimension très importante pour les entreprises au Chili, et que la préoccupation pour l'identité - image - réputation de marque corporate est essentielle et se situe au cœur de ce qu'ils désignent comme modèle d'affaires.

Finalement, par rapport à la tendance antérieure de gestion de la marque, il convient à présent de prendre en considération la forte présence de la dimension communicationnelle. Dans notre étude portant de la stratégie de communication de la marque dans les entreprises chiliennes, il apparaît que huit principales tendances communicationnelles sont identifiées. Parmi elles, les entreprises sont, en premier lieu, préoccupées de l'Identité - Image - Réputation Corporative ; et en second lieu, du Co-branding ; suivi respectivement du e-Branding, du Brand Experience, du Social Branding ou Responsabilité Sociale de l'Entreprise, du Green Branding, du Branding Mutante, et de l'Antibranding. Ces résultats montrent à quel point les entreprises chiliennes développent les mêmes stratégies de communication que partout ailleurs dans le monde et soulignent le développement de la fonction Communication en cette partie du monde.

\section{BIBLIOGRAPHIE}

SALEH Felipe, «El nuevo orden en la "industria" de la comunicación estratégica». El Mostrador.cl Consultado el 31 de mayo. http://www.elmostrador.cl/noticias/ pais/2010/05/31/el-nuevo-orden-en-la-\%e2\%80\%9cindustria\%e2\%80\%9d-de-lacomunicacion-estrategica, 2010.

HERRERA Raúl, "Crecimiento y desarrollo de la industria de la comunicación estratégica en Chile.» Revue Chasqui, número 108, 2010. Herrera Raúl, Etude: Estado 
del Arte de la Comunicación Corporativa en Chile, 2008. Carrasco Eduardo: Marcas en la Cabeza. Catalonia, 2009.

ÁLVAREZ Pedro, Chile Marca Registrada. Ocho Libros Editores, 2008.

TIRONI Eugenio y CAVALLO Ascanio, Comunicación Estratégica. Taurus, 2004.

COSTA Joan, Imagen Corporativa en el siglo XXI. La Crujía, 2003.

CAPPRIOTTI Paul, Branding Corporativo. Colección Libros de la Empresa, 2009.

CAPPRIOTTI Paul, Gestión de Marca Corporativa. La Crujía, 2007.

MAS Francisco, Estudio: Tesina de Suficiencia Doctoral. Universidad de Málaga. España, 2010.

Résumé : Il s'agit ici de présenter la question de la stratégie de communication dans les entreprises chiliennes autant dans sa dimension interne que sa dimension corporate liée à la gestion des stakeholders externes. À la lumière du passé et du présent, nous analyserons les tendances de stratégies de communication dans la gestion de marque que les entreprises chiliennes appliquent afin de s'adresser à leurs publics divers. Dans ce domaine, un fait saillant concerne les méthodes de gestion des actifs symboliques comme par exemple la gestion de la marque en tant qu'identité- image-réputation, le Green Branding, le co ou social Branding etc. Ce travail repose sur des exemples représentatifs qui permettent de comprendre les différents niveaux et stratégies de développement de ces dimensions de la stratégie de communication.

Mots-clé : Stratégie de communication, communication corporative, marque, branding, identité, image, réputation corporative

Abstract : This is a study on the status of strategic communication in Chilean companies,
in reference both to the internal strategic communication and its corporative dimension, and
to communication management for external stakeholders. From these verifications, trends of
strategic communication in brand management applied by the companies in Chile are also
reviewed so as to dialogue with its several audiences. In this scope, varied devices stand out
in regards to the management methods of these symbolic assets, including: Brand management
as to Identity - Image - Corporate Reputation; Green Branding; Co or Social Branding etc.
Representative samples are collected in this work, which allow to understand the different levels
and strategies of these dimensions' roll-out in strategic communication.

Keywords: Strategic Communication, Corporate Communication, Brand, Branding, Identity, Image, Corporate Reputation. 